PROCEEDINGS OF THE

AMERICAN MATHEMATICAL SOCIETY

Volume 139, Number 5, May 2011, Pages 1805-1817

S 0002-9939(2010)10615-9

Article electronically published on November 1, 2010

\title{
THE RADIAL DEFOCUSING ENERGY-SUPERCRITICAL NONLINEAR WAVE EQUATION IN ALL SPACE DIMENSIONS
}

\author{
ROWAN KILLIP AND MONICA VISAN
}

(Communicated by Hart F. Smith)

\begin{abstract}
We consider the defocusing nonlinear wave equation $u_{t t}-\Delta u+$ $|u|^{p} u=0$ with spherically-symmetric initial data in the regime $\frac{4}{d-2}<p<\frac{4}{d-3}$ (which is energy-supercritical) and dimensions $3 \leq d \leq 6$; we also consider $d \geq 7$, but for a smaller range of $p>\frac{4}{d-2}$. The principal result is that blowup (or failure to scatter) must be accompanied by blowup of the critical Sobolev norm. An equivalent formulation is that maximal-lifespan solutions with bounded critical Sobolev norm are global and scatter.
\end{abstract}

\section{INTRODUCTION}

We consider the initial value problem for the defocusing nonlinear wave equation in $d \geq 3$ space dimensions:

$$
\left\{\begin{array}{l}
u_{t t}-\Delta u+F(u)=0, \\
u(0)=u_{0}, u_{t}(0)=u_{1},
\end{array}\right.
$$

where the nonlinearity $F(u)=|u|^{p} u$ is energy-supercritical, that is, $p>\frac{4}{d-2}$.

The class of solutions to (1.1) is left invariant by the scaling

$$
u(t, x) \mapsto \lambda^{\frac{2}{p}} u(\lambda t, \lambda x),
$$

which determines the critical Sobolev space for initial data, namely, $\left(u_{0}, u_{1}\right) \in$ $\dot{H}_{x}^{s_{c}} \times \dot{H}_{x}^{s_{c}-1}$, where the critical regularity is $s_{c}:=\frac{d}{2}-\frac{2}{p}$. Notice that $p>\frac{4}{d-2}$ precisely corresponds to $s_{c}>1$; since the energy

$$
E(u)=\int_{\mathbb{R}^{d}}\left(\frac{1}{2}\left|u_{t}\right|^{2}+\frac{1}{2}|\nabla u|^{2}+\frac{1}{p+2}|u|^{p+2}\right) d x
$$

scales like $s=1$, this regime is known as energy-supercritical.

Let us start by making the notion of a solution more precise.

Definition 1.1 (Solution). A function $u: I \times \mathbb{R}^{d} \rightarrow \mathbb{R}$ on an open time interval $0 \in I \subset \mathbb{R}$ is a (strong) solution to (1.1) if $\left(u, u_{t}\right) \in C_{t}^{0}\left(K ; \dot{H}_{x}^{s_{c}} \times \dot{H}_{x}^{s_{c}-1}\right)$ and

Received by the editors February 8, 2010 and, in revised form, May 26, 2010.

2010 Mathematics Subject Classification. Primary 35L71.

The first author was supported by NSF grant DMS-0701085.

The second author was supported by NSF grant DMS-0901166.

(C)2010 American Mathematical Society Reverts to public domain 28 years from publication 
$u \in L_{t, x}^{(d+1) p / 2}\left(K \times \mathbb{R}^{d}\right)$ for all compact $K \subset I$, and obeys the Duhamel formula

$$
\begin{aligned}
{\left[\begin{array}{c}
u(t) \\
u_{t}(t)
\end{array}\right]=} & {\left[\begin{array}{cc}
\cos (t|\nabla|) & |\nabla|^{-1} \sin (t|\nabla|) \\
-|\nabla| \sin (t|\nabla|) & \cos (t|\nabla|)
\end{array}\right]\left[\begin{array}{c}
u(0) \\
u_{t}(0)
\end{array}\right] } \\
& -\int_{0}^{t}\left[\begin{array}{c}
|\nabla|^{-1} \sin ((t-s)|\nabla|) \\
\cos ((t-s)|\nabla|)
\end{array}\right] F(u(s)) d s
\end{aligned}
$$

for all $t \in I$. We refer to the interval $I$ as the lifespan of $u$. We say that $u$ is a maximal-lifespan solution if the solution cannot be extended to any strictly larger interval. We say that $u$ is a global solution if $I=\mathbb{R}$.

We define the scattering size of a solution to (1.1) on a time interval $I$ by

$$
\|u\|_{S(I)}:=\left(\int_{I} \int_{\mathbb{R}^{d}}|u(t, x)|^{\frac{(d+1) p}{2}} d x d t\right)^{\frac{2}{(d+1) p}}
$$

Associated to the notion of a solution is a corresponding notion of blowup. By the standard local theory, the following precisely corresponds to the impossibility of continuing the solution in a manner consistent with Definition 1.1 .

Definition 1.2 (Blowup). We say that a solution $u$ to (1.1) blows up forward in time if there exists a time $t_{1} \in I$ such that

$$
\|u\|_{S\left(\left[t_{1}, \sup I\right)\right)}=\infty
$$

and that $u$ blows up backward in time if there exists a time $t_{1} \in I$ such that

$$
\|u\|_{S\left(\left(\inf I, t_{1}\right]\right)}=\infty \text {. }
$$

Our purpose here is to give a short proof of the following result:

Theorem 1.3 (Spacetime bounds). Assume that $\frac{4}{d-2}<p<\frac{4}{d-3}$ for $3 \leq d \leq 6$ and that $\frac{4}{d-2}<p<\frac{d(d-1)-\sqrt{d^{2}(d-1)^{2}-16(d+1)^{2}}}{2(d+1)}$ if $d \geq 7$. Let $u: I \times \mathbb{R}^{d} \rightarrow \mathbb{R}$ be a spherically-symmetric solution to (1.1) such that $\left(u, u_{t}\right) \in L_{t}^{\infty}\left(I ; \dot{H}_{x}^{s_{c}} \times \dot{H}_{x}^{s_{c}-1}\right)$. Then

$$
\|u\|_{S(I)} \leq C\left(\left\|\left(u, u_{t}\right)\right\|_{L_{t}^{\infty}\left(I ; \dot{H}_{x}^{s_{c}} \times \dot{H}_{x}^{s_{c}-1}\right)}\right) .
$$

For $d=3$ this was proved by Kenig and Merle [13. In [19] we proved this result for nonradial data. While preparing [19, we realized that it is possible to give a short proof of Theorem 1.3 that works uniformly in all dimensions. That is the topic of this paper. In keeping with our goal of a simple presentation, we have restricted ourselves to the specific values of $p$ stated in the theorem. These hypotheses represent the combination of two restrictions, one related to the local theory (which dominates in high dimensions) and another dictated by the Morawetz inequality $\left(s_{c}<3 / 2\right)$. The former restriction stems from our desire to present as simple and uniform a local theory as possible. While one may certainly obtain a larger range of $p$ in this setting by some piecemeal approach, it is not clear to us how to obtain the full range dictated by our principal hypothesis $s_{c}<3 / 2$. Indeed, note that one natural restriction is the smoothness condition $s_{c}<p+1$; this allows us to take $s_{c}$ derivatives of the nonlinearity. Our condition for $d \geq 7$ is equivalent to $s_{c}<p+1-\left(\frac{1}{d+1}+\frac{p}{2}\right)$.

In low dimensions, the sole restriction on $p$ is $p<\frac{4}{d-3}$ and corresponds to $s_{c}<3 / 2$, which nevertheless covers all values of $p$ when $d=3$. This condition is 
dictated by the Morawetz inequality. The methods presented here do not immediately extend to higher values of $p$. The problem arises in Section 4 and could be circumvented by proving that the almost periodic solutions discussed below actually lie in $L_{t}^{\infty}\left(\dot{H}_{x}^{s} \times \dot{H}_{x}^{s-1}\right)$ for some $s<3 / 2$. Arguments showing how this can be done (even in the nonradial setting) may be found in [15, 17, 19]; however, this is significantly more involved than what we chose to present here.

Since completion of this work, Bulut 4 has treated the cubic nonlinearity (with nonradial initial data) in dimensions $d \geq 6$ by employing the strategy from [15, 17.

As mentioned above, finite-time blowup of a solution to (1.1) must be accompanied by divergence of the scattering size defined in (1.5). Thus, Theorem 1.3 immediately implies

Corollary 1.4 (Spacetime bounds). If $u: I \times \mathbb{R}^{d} \rightarrow \mathbb{R}$ is a maximal-lifespan spherically-symmetric solution to (1.1) with $\left(u, u_{t}\right) \in L_{t}^{\infty}\left(I ; \dot{H}_{x}^{s_{c}} \times \dot{H}_{x}^{s_{c}-1}\right)$, then $u$ is global,

$$
\|u\|_{S(\mathbb{R})} \leq C\left(\left\|\left(u, u_{t}\right)\right\|_{L_{t}^{\infty}\left(\mathbb{R} ; \dot{H}_{x}^{s_{c}} \times \dot{H}_{x}^{s_{c}-1}\right)}\right),
$$

and $u$ scatters in the sense that

$$
\left\|\left(u, u_{t}\right)-\left(u^{ \pm}, u_{t}^{ \pm}\right)\right\|_{\dot{H}_{x}^{s c} \times \dot{H}_{x}^{s_{c}-1}} \rightarrow 0 \quad \text { as } \quad t \rightarrow \pm \infty,
$$

for two solutions $u^{ \pm}$of the linear wave equation.

This corollary takes on a more appealing form if we rephrase it in the contrapositive:

Corollary 1.5 (Nature of blowup). A spherically-symmetric solution $u: I \times \mathbb{R}^{d} \rightarrow$ $\mathbb{R}$ to (1.1) can only blow up in finite time or be global but fail to scatter if its $\dot{H}_{x}^{s_{c}} \times \dot{H}_{x}^{s_{c}-1}$ norm diverges.

When $p=\frac{4}{d-2}$, or equivalently, $s_{c}=1$, the critical Sobolev norm is automatically bounded in time by virtue of the conservation of energy. This energy-critical case of (1.1) has received particular attention because of this property. Global wellposedness was proved in a series of works $7,8,2,9,24,28,25,26$ with finiteness of the scattering size being added later; see [1, 6, 22, 23, 30. Certain monotonicity formulae, namely the Morawetz and energy flux identities, play an important role in all these results. It is important that these monotonicity formulae also have critical scaling.

In the energy-supercritical case discussed in this paper, all conservation laws and monotonicity formulae have scaling below the critical regularity. At the present moment, there is no technology for treating large-data dispersive equations without some a priori control of a critical norm. This is the purpose of the $L_{t}^{\infty}\left(I ; \dot{H}_{x}^{s_{c}} \times\right.$ $\dot{H}_{x}^{s_{c}-1}$ ) assumption in Theorem 1.3, it plays the role of the missing conservation law at the critical regularity. To deal with the fact that the basic monotonicity formula scales like the energy (rather than the critical regularity), we employ a space truncation in the manner of [2]; see also [18, 29].

1.1. Outline of the proof. We argue by contradiction. By the fundamental observations of Keraani [14] and Kenig-Merle [11, we know that failure of Theorem 1.3 guarantees the existence of certain minimal counterexamples and, moreover, such solutions have good compactness properties. These properties are best described in terms of the following notion: 
Definition 1.6 (Almost periodicity modulo scaling). A solution $u$ to (1.1) with lifespan $I$ is said to be almost periodic modulo scaling if $\left(u, u_{t}\right)$ is bounded in $\dot{H}_{x}^{s_{c}} \times \dot{H}_{x}^{s_{c}-1}$ and there exist functions $N: I \rightarrow \mathbb{R}^{+}$and $C: \mathbb{R}^{+} \rightarrow \mathbb{R}^{+}$such that for all $t \in I$ and $\eta>0$,

$$
\left.\left.\int_{|x| \geq C(\eta) / N(t)}|| \nabla\right|^{s_{c}} u(t, x)\right|^{2} d x+\left.\left.\int_{|x| \geq C(\eta) / N(t)}|| \nabla\right|^{s_{c}-1} u_{t}(t, x)\right|^{2} d x \leq \eta
$$

and

$$
\int_{|\xi| \geq C(\eta) N(t)}|\xi|^{2 s_{c}}|\hat{u}(t, \xi)|^{2} d \xi+\int_{|\xi| \geq C(\eta) N(t)}|\xi|^{2\left(s_{c}-1\right)}\left|\hat{u}_{t}(t, \xi)\right|^{2} d \xi \leq \eta .
$$

We refer to the function $N(t)$ as the frequency scale function for the solution $u$ and to $C(\eta)$ as the compactness modulus function.

Remarks. 1. Spherical symmetry forces the bulk of the solution to concentrate around the spatial origin. This is the reason for the absence of a spatial center function $x(t)$.

2. The continuous image of a compact set is compact. Thus, by Sobolev embedding, almost periodic (modulo scaling) solutions obey the following: For each $\eta>0$ there exists $C(\eta)>0$ so that

$$
\|u(t, x)\|_{L_{t}^{\infty} L_{x}^{\frac{d p}{2}}(\{|x| \geq C(\eta) / N(t)\})}+\left\|\nabla_{t, x} u(t, x)\right\|_{L_{t}^{\infty} L_{x}^{\frac{d p}{p+2}}(\{|x| \geq C(\eta) / N(t)\})} \leq \eta,
$$

where $\nabla_{t, x} u=\left(u_{t}, \nabla u\right)$ denotes the space-time gradient of $u$.

With these preliminaries out of the way, we can now describe the first major milestone in the proof of Theorem 1.3 .

Theorem 1.7 (Three special scenarios for blowup). Suppose that Theorem 1.3 failed. Then there exists a maximal-lifespan spherically-symmetric solution $u: I \times$ $\mathbb{R}^{d} \rightarrow \mathbb{R}$, which obeys $\left(u, u_{t}\right) \in L_{t}^{\infty}\left(I ; \dot{H}_{x}^{s_{c}} \times \dot{H}_{x}^{s_{c}-1}\right)$, is almost periodic modulo scaling, and $\|u\|_{S(I)}=\infty$. Moreover, we can also ensure that the lifespan $I$ and the frequency scale function $N: I \rightarrow \mathbb{R}^{+}$match one of the following three scenarios:

I. (Finite-time blowup) We have that either $\sup I<\infty$ or $|\inf I|<\infty$.

II. (Soliton-like solution) We have $I=\mathbb{R}$ and $N(t)=1$ for all $t \in \mathbb{R}$.

III. (Low-to-high frequency cascade) We have $I=\mathbb{R}$,

$$
\inf _{t \in \mathbb{R}} N(t) \geq 1, \quad \text { and } \quad \limsup _{t \rightarrow+\infty} N(t)=\infty .
$$

The proof of Theorem 1.7 follows a well-travelled path. See [16 for an introduction to these techniques including two worked examples and references up to that time. Let us briefly review the ingredients: (i) A concentration compactness principle (= profile decomposition) for the linear propagator. The very first example of this was worked out for the wave equation (with $d=3$ ) in 1]. The extension to all dimensions can be found in 3 . (ii) A perturbation theory for the nonlinear equation. While the basic framework is standard, each equation has its peculiarities, particularly when small-power nonlinearities are involved. We discuss this at some length in Section 3 in part because our arguments unify and simplify existing results for certain special cases. (iii) A decoupling argument. This is usually fairly direct; however, some subtleties arise in the model discussed in this paper due to the fact that $s_{c}>1$ and $p$ is small. The requisite technology can be found in [17. 
With Theorem 1.7 in hand, the proof of Theorem 1.3 reduces to showing that none of the three special scenarios can occur. In Section 5 we show that the first scenario, a finite-time blow-up solution, cannot exist because it is inconsistent with the conservation of energy. In Section 4 we show that neither of the other two scenarios can occur, since they are inconsistent with the (truncated) Morawetz identity (cf. Lemma 2.5) when $p<\frac{4}{d-3}$.

\section{NLW BACKGROUND}

We write $X \lesssim Y$ to indicate that $X \leq C Y$ for some dimension-dependent constant $C$, which may change from line to line. Other dependencies will be indicated with subscripts, for example, $X \lesssim_{u} Y$.

2.1. Strichartz estimates. One of the most fundamental tools in the modern analysis of nonlinear wave equations is the Strichartz estimate. We record some particular instances of this estimate below. For further information, see [10, 23, 27] and the references therein.

Definition 2.1 (Admissible pairs). We say that the pair $(q, r)$ is wave-admissible if

$$
\frac{1}{q}+\frac{d-1}{2 r} \leq \frac{d-1}{4}, \quad 2 \leq q \leq \infty, \quad \text { and } \quad 2 \leq r<\infty .
$$

Lemma 2.2 (Strichartz estimates). Fix $d \geq 3$. Let I be a compact time interval and let $u: I \times \mathbb{R}^{d} \rightarrow \mathbb{R}$ be a solution to the forced wave equation

$$
u_{t t}-\Delta u+F=0 .
$$

Then for any $t_{0} \in I$ and any wave-admissible pair $(q, r)$,

$$
\begin{aligned}
\left\|\nabla_{t, x} u\right\|_{L_{t}^{\infty} \dot{H}_{x}^{s_{c}-1}} & +\|u\|_{S(I)}+\left\||\nabla|^{s_{c}-\frac{1}{2}} u\right\|_{L_{t, x}^{\frac{2(d+1)}{d-1}}}+\left\||\nabla|^{\gamma-1} \nabla_{t, x} u\right\|_{L_{t}^{q} L_{x}^{r}} \\
& \lesssim\left\|\nabla_{t, x} u\left(t_{0}\right)\right\|_{\dot{H}_{x}^{s_{c}-1}}+\left\||\nabla|^{s_{c}-\frac{1}{2}} F\right\|_{L_{t, x}^{\frac{2(d+1)}{d+3}}},
\end{aligned}
$$

provided $\frac{1}{q}+\frac{d}{r}=\frac{2}{p}+\gamma$. All spacetime norms in the formula above are on $I \times \mathbb{R}^{d}$.

We will use the notation

$$
\|u\|_{S^{s_{c}(I)}}:=\sup \left\||\nabla|^{\gamma-1} \nabla_{t, x} u\right\|_{L_{t}^{q} L_{x}^{r}}
$$

where the supremum is taken over all admissible pairs $(q, r)$ and numbers $\gamma$ obeying the scaling condition $\frac{1}{q}+\frac{d}{r}=\frac{2}{p}+\gamma$, with $r \leq r_{*}(d, p)$ dictated by the largest exponent appearing in the arguments below.

The following result will be needed in Section 3, its proof requires only minor modifications to the proof of the Christ-Weinstein fractional chain rule presented in [31, $\S 2.5]$.

Lemma 2.3 (Derivatives of differences). Let $F(u)=|u|^{p} u$ with $p>0$ and let $0<s<1$. Then for $1<q, q_{1}, q_{2}<\infty$ such that $\frac{1}{q}=\frac{1}{q_{1}}+\frac{p}{q_{2}}$, we have

$$
\left\||\nabla|^{s}[F(u+v)-F(u)]\right\|_{q} \lesssim\left\||\nabla|^{s} u\right\|_{q_{1}}\|v\|_{q_{2}}^{p}+\left\||\nabla|^{s} v\right\|_{q_{1}}\|u+v\|_{q_{2}}^{p} .
$$


Proof. By the Fundamental Theorem of Calculus,

$$
\begin{aligned}
|F(u(x))-F(u(y))| & =|u(x)-u(y)|\left|\int_{0}^{1} F^{\prime}(u(y)+t[u(x)-u(y)]) d t\right| \\
& \lesssim_{p}|u(x)-u(y)|\left\{|u(x)|^{p}+|u(y)|^{p}\right\},
\end{aligned}
$$

and similarly for $F(u+v)$. Combining these gives

$$
\begin{aligned}
\mid[F(u+v)-F(u)](x) & -[F(u+v)-F(u)](y) \mid \\
& \lesssim\{|v(x)-v(y)|+|u(x)-u(y)|\}\{H(x)+H(y)\},
\end{aligned}
$$

where $H(x)=|u(x)|^{p}+|v(x)|^{p}$. With this estimate in hand, one may now follow verbatim the arguments used to prove Proposition 5.1 in [31, §2.5]. More precisely, this estimate is used in place of (5.4) in that book.

As $p$ may be less than one, we will also need a version of the fractional chain rule for fractional powers:

Lemma 2.4 (Fractional chain rule for a Hölder continuous function, 32]). Suppose $G$ is a Hölder continuous function of order $0<p<1$. Then, for every $0<s<p$, $1<q<\infty$, and $\frac{s}{p}<\sigma<1$ we have

$$
\left\||\nabla|^{s} G(u)\right\|_{q} \lesssim\left\||u|^{p-\frac{s}{\sigma}}\right\|_{q_{1}}\left\||\nabla|^{\sigma} u\right\|_{\frac{s}{\sigma} q_{2}}^{\frac{s}{\sigma}},
$$

provided $\frac{1}{q}=\frac{1}{q_{1}}+\frac{1}{q_{2}}$ and $\left(1-\frac{s}{p \sigma}\right) q_{1}>1$.

2.2. Morawetz inequality. Next we derive a space-localized Morawetz identity (cf. 20, 21]). It is a very close analogue of the formula used in 2] in the NLS setting; see also [18, 29].

Lemma 2.5 (Space-localized Morawetz). Let $u: I \times \mathbb{R}^{d} \rightarrow \mathbb{R}$ be a solution of (1.1) with $d \geq 3$. Then

$$
\int_{I} \int_{|x| \leq|I|} \frac{|u(t, x)|^{p+2}}{|x|} d x d t \lesssim|I|^{d-2-\frac{4}{p}}\left(\left\|\nabla_{t, x} u\right\|_{L_{t}^{\infty} \dot{H}_{x}^{s_{c}-1}}^{2}+\left\|\nabla_{t, x} u\right\|_{L_{t}^{\infty} \dot{H}_{x}^{s_{c}-1}}^{p+2}\right) .
$$

Proof. By direct computation, one sees that taking the time derivative of

$$
M(t):=\int_{\mathbb{R}^{3}}\left(-a_{j}(x) u_{t}(t, x) u_{j}(t, x)-\frac{1}{2} a_{j j}(x) u(t, x) u_{t}(t, x)\right) d x
$$

yields

$$
\frac{d M}{d t}=\int_{\mathbb{R}^{3}}\left(a_{j k}(x) u_{j}(t, x) u_{k}(t, x)+\frac{p}{2(p+2)} a_{j j}(x) u(t, x)^{p+2}-\frac{1}{4} a_{j j k k}(x) u(t, x)^{2}\right) d x
$$

for general functions $a: \mathbb{R}^{d} \rightarrow \mathbb{R}$. Here subscripts denote spatial derivatives and repeated indices are summed. Setting $R=|I|$, we choose $a(x):=R \psi(|x| / R)$, where $\psi(r)$ is a smooth nondecreasing function obeying $\psi(r)=r$ if $r \leq 1$ and $\psi(r)=3 / 2$ when $r \geq 2$.

Simple computations show that for $|x| \leq R$, we have

$$
a_{k}(x)=\frac{x_{k}}{|x|}, \quad a_{j j}(x)=\frac{d-1}{|x|}>0, \quad-a_{j j k k}(x)>0 \quad \text { (as a distribution), }
$$

and the matrix $a_{j k}(x)$ is positive definite. On the other hand, when $R \leq|x| \leq 2 R$,

$$
\left|a_{k}(x)\right| \lesssim 1, \quad\left|a_{j k}(x)\right| \lesssim R^{-1}, \quad \text { and } \quad\left|-a_{j j k k}(x)\right| \lesssim R^{-3}
$$


while all derivatives of $a$ vanish when $|x| \geq 2 R$. Combining this information with Hölder's, Hardy's, and Sobolev's inequalities yields

$$
|M(t)| \lesssim R^{d-2-\frac{4}{p}}\left\|\nabla_{t, x} u\right\|_{L_{t}^{\infty} L_{x}^{\frac{d p}{p+2}}}^{2} \lesssim R^{d-2-\frac{4}{p}}\left\|\nabla_{t, x} u\right\|_{L_{t}^{\infty} \dot{H}_{x}^{s_{c}-1}}^{2}
$$

and similarly,

$\frac{d M}{d t} \geq \frac{p(d-1)}{2(p+2)} \int_{|x| \leq R} \frac{|u(t, x)|^{p+2}}{|x|} d x-R^{d-3-\frac{4}{p}} O\left(\left\|\nabla_{t, x} u\right\|_{L_{t}^{\infty} \dot{H}_{x}^{s_{c}-1}}^{2}+\left\|\nabla_{t, x} u\right\|_{L_{t}^{\infty} \dot{H}_{x}^{s_{c}-1}}^{p+2}\right)$.

The result now follows by the Fundamental Theorem of Calculus.

2.3. Potential energy concentration. To make use of Lemma 2.5, we need a lower bound on the left-hand side. This cannot be done pointwise in time, but we do have the following:

Proposition 2.6 (Potential energy concentration). Let $u$ be a global solution to (1.1) that is almost periodic modulo scaling. Then, there exists $C=C(u)$ so that

$$
\int_{I} \int_{|x-x(t)| \leq C / N(t)} N(t)|u(t, x)|^{p+2} d x d t \gtrsim u \int_{I} N(t)^{\frac{4}{p}-(d-3)} d t,
$$

uniformly for all intervals $I=\left[t_{1}, t_{2}\right] \subseteq \mathbb{R}$ with $t_{2} \geq t_{1}+N\left(t_{1}\right)^{-1}$.

Proof. Without the additional factor of $N(t)$ on the left (and so also on the right), this is proved in [19]; see Corollary 3.5 there. However, the very first step in that proof is to split $I$ into intervals where $N(t)$ is essentially constant. For this reason, (2.2) also follows from the argument presented there.

\section{Stability}

In this section we sketch the proof of the stability result for (1.1), the only part of the proof of Theorem 1.7 that is not already in the literature. We note here that the proof works equally well in the defocusing and focusing cases.

Theorem 3.1 (Stability). Assume that $\frac{4}{d-2} \leq p<\frac{4}{d-3}$ if $3 \leq d \leq 6$ and assume that $\frac{4}{d-2} \leq p<\frac{d(d-1)-\sqrt{d^{2}(d-1)^{2}-16(d+1)^{2}}}{2(d+1)}$ if $d \geq 7$. Let I a compact time interval containing zero and let $\tilde{u}$ be an approximate solution to (1.1) on $I \times \mathbb{R}^{d}$ in the sense that

$$
\tilde{u}_{t t}-\Delta \tilde{u}+F(\tilde{u})+e=0
$$

for some function e. Assume that

$$
\begin{aligned}
\left\|\nabla_{t, x} \tilde{u}\right\|_{L_{t}^{\infty} \dot{H}_{x}^{s_{c}-1}\left(I \times \mathbb{R}^{d}\right)} & \leq M, \\
\|\tilde{u}\|_{S(I)} & \leq L
\end{aligned}
$$

for some positive constants $M$ and L. Let $\left(u_{0}, u_{1}\right) \in \dot{H}_{x}^{s_{c}} \times \dot{H}_{x}^{s_{c}-1}$ be such that

$$
\left\|\left(u_{0}, u_{1}\right)-\left(\tilde{u}_{0}, \tilde{u}_{1}\right)\right\|_{\dot{H}_{x}^{s_{c}} \times \dot{H}_{x}^{s_{c}-1}} \leq \varepsilon
$$

and suppose also that the error e obeys

$$
\left\||\nabla|^{s_{c}-1 / 2} e\right\|_{L_{t, x}^{\frac{2(d+1)}{d+3}}\left(I \times \mathbb{R}^{d}\right)} \leq \varepsilon
$$


for some $0<\varepsilon<\varepsilon_{1}=\varepsilon_{1}(M, L)$. Then, there exists a unique strong solution $u: I \times \mathbb{R}^{d} \mapsto \mathbb{R}$ to (1.1) with initial data $\left(u_{0}, u_{1}\right)$ at time $t=0$; moreover,

$$
\begin{aligned}
\|u-\tilde{u}\|_{S(I)} & \leq C(M, L) \varepsilon^{c}, \\
\|u-\tilde{u}\|_{S^{s_{c}(I)}} & \leq C(M, L), \\
\|u\|_{S^{s(I)}} & \leq C(M, L),
\end{aligned}
$$

where $c$ is a positive constant that depends on $d, p, M$, and $L$.

The general strategy for proving stability for a dispersive equation is by now standard and is reviewed along with references in [16. Indeed, special cases of Theorem 3.1 have appeared before; see [5, 12, 13]. Nevertheless, there is some flexibility in the method in terms of which spaces one chooses to work in and we contend that we provide a simpler treatment of the existing results just mentioned.

As in our previous work on the nonlinear Schrödinger equation [16, 17, we will work in spaces with a small fractional number of derivatives. We close our bootstrap in the following spaces:

$$
\|u\|_{X(I)}:=\left\||\nabla|^{s_{c}-\frac{1}{2}} u\right\|_{L_{t, x}^{\frac{2(d+1)}{d-1}}\left(I \times \mathbb{R}^{d}\right)} \text { and }\|F\|_{Y(I)}:=\left\||\nabla|^{s_{c}-\frac{1}{2}} F\right\|_{L_{t, x}^{\frac{2(d+1)}{d+3}}\left(I \times \mathbb{R}^{d}\right)}
$$

when $3 \leq d \leq 6$, and when $d \geq 7$,

$$
\begin{aligned}
\|u\|_{X(I)} & :=\left\||\nabla|^{p / 2} u\right\|_{L_{t}^{\frac{4(d+1)+p^{2}(d+1)-p d(d-1)}{4}} L_{x}^{\frac{2(d+1)}{d-1}}} \\
\|F\|_{Y(I)} & :=\left\||\nabla|^{p / 2} F\right\|_{L_{t}^{\frac{4(d+1)+p^{2}(d+1)-p\left(d^{2}-d-4\right)}{4(d+1)}} L_{x}^{\frac{2(d+1)}{d+3}}\left(I \times \mathbb{R}^{d}\right)} .
\end{aligned}
$$

The additional restriction on $p$ when $d \geq 7$ is to ensure that the time exponent in the definition of $X$ (and so also $Y$ ) is positive (and finite).

The space $X(I)$ in which the solution will be measured is related to the space $Y(I)$ in which the nonlinearity will be measured via a Strichartz-type inequality:

Lemma 3.2 (A Strichartz-type inequality).

$$
\left\|\int_{0}^{t} \frac{\sin ((t-s)|\nabla|)}{|\nabla|} F(s) d s\right\|_{X(I)} \lesssim\|F\|_{Y(I)} .
$$

Proof. In dimensions $3 \leq d \leq 6$, this is an instance of the usual Strichartz inequality (cf. Lemma 2.2), while for $d \geq 7$ it is one of the standard exotic Strichartz estimates. The proof is simple and the same in either case; we review it below.

As noted for example in [26, §4.3], the wave propagator obeys the frequencylocalized dispersive estimate

$$
\left\|\frac{\sin ((t-s)|\nabla|)}{|\nabla|} P_{N} f\right\|_{L_{x}^{\frac{2(d+1)}{d-1}}} \lesssim|t-s|^{-\frac{d-1}{d+1}}\left\|P_{N} f\right\|_{L_{x}^{\frac{2(d+1)}{d+3}}},
$$

from which the result follows by the Hardy-Littlewood-Sobolev inequality and elementary Littlewood-Paley theory.

We also note the following relations between the various spaces involved: 
Lemma 3.3. With $S$ as in (1.5) and $S^{s_{c}}$ as in (2.1),

$$
\begin{aligned}
\|u\|_{X(I)} & \leq\|u\|_{S^{s_{c}(I)}}, \\
\|u\|_{S(I)} & \leq\|u\|_{X(I)}^{\theta}\|u\|_{S^{s_{c}(I)}}^{1-\theta} \quad \text { for some } \quad 0<\theta<1, \\
\|u\|_{X(I)} & \leq\|u\|_{S(I)}^{\tilde{\theta}}\|u\|_{S^{s_{c}(I)}}^{1-\tilde{\theta}} \quad \text { for some } \quad 0<\tilde{\theta}<1, \\
\|F(u)\|_{Y(I)} & \lesssim\|u\|_{X(I)}\|u\|_{S(I)}^{p} \lesssim\|u\|_{X(I)}^{1+\theta p}\|u\|_{S^{s(I)}}^{(1-\theta) p} .
\end{aligned}
$$

Our last estimates are for differences of nonlinearities. For $3 \leq d \leq 6$,

$$
\begin{aligned}
\| F(u)- & F(\tilde{u}) \|_{Y(I)} \\
& \lesssim\|\tilde{u}\|_{X(I)}\|u-\tilde{u}\|_{S(I)}^{p}+\|u-\tilde{u}\|_{X(I)}\left\{\|\tilde{u}\|_{S(I)}^{p}+\|u-\tilde{u}\|_{S(I)}^{p}\right\},
\end{aligned}
$$

while for $d \geq 7$ we need

$$
\begin{aligned}
\| F(u)- & F(\tilde{u}) \|_{Y(I)} \\
& \lesssim\|u-\tilde{u}\|_{X(I)}\left\{\|u-\tilde{u}\|_{S^{s_{c}(I)}}^{p-\frac{p}{d}}\|u-\tilde{u}\|_{S(I)}^{\frac{p}{d}}+\|\tilde{u}\|_{S^{c_{c}(I)}}^{p-\frac{p}{d}}\|\tilde{u}\|_{S(I)}^{\frac{p}{d}}\right\},
\end{aligned}
$$

as well as the direct analogue of (3.8), namely,

$$
\begin{aligned}
\||\nabla|^{s_{c}-\frac{1}{2}} & {[F(u)-F(\tilde{u})] \|_{L_{t, x}^{\frac{2(d+1)}{d+3}}\left(I \times \mathbb{R}^{d}\right)} } \\
& \lesssim\|\tilde{u}\|_{S^{s_{c}(I)}}\|u-\tilde{u}\|_{S(I)}^{p}+\|u-\tilde{u}\|_{S^{s_{c}(I)}}\left\{\|\tilde{u}\|_{S(I)}^{p}+\|u-\tilde{u}\|_{S(I)}^{p}\right\} .
\end{aligned}
$$

Proof. The first four estimates follow from straightforward applications of Sobolev embedding, interpolation, Hölder's inequality, together with the fractional chain rule Lemma 2.4.

The inequalities (3.8) and (3.10) are consequences of Lemma 2.3 and Hölder's inequality.

To derive (3.9), one first uses the Fundamental Theorem of Calculus to write

$$
F(u)-F(\tilde{u})=(u-\tilde{u}) \int_{0}^{1} F^{\prime}(\tilde{u}+\tau[u-\tilde{u}]) d \tau
$$

and thence, via the fractional product rule (and Sobolev embedding),

$$
\|F(u)-F(\tilde{u})\|_{Y(I)} \lesssim\|u-\tilde{u}\|_{X(I)} \sup _{\tau \in[0,1]}\left\||\nabla|^{p / 2} F^{\prime}(\tilde{u}+\tau[u-\tilde{u}])\right\|_{L_{t}^{\frac{d+1}{2}} \frac{2 d(d+1)}{L_{x}^{4 d+p(d+1)}}} .
$$

The estimate (3.9) now follows from Lemma 2.4, in which we take $\sigma=\frac{d}{2(d-1)}$.

With these preliminaries out of the way, we are ready to resume the proof of Theorem 3.1. For the remainder of the proof, all spacetime norms are over $I \times \mathbb{R}^{d}$. By standard iterative arguments (and subdividing the original time interval), it suffices to prove the claim with hypothesis (3.2) replaced by

$$
\|\tilde{u}\|_{S(I)} \leq \delta
$$

where $\delta$ is a small constant allowed to depend on $M$. By Lemma 3.3 , we see that one can transfer bounds (and smallness) between the $X$ and $S$ norms; thus, it suffices to prove the claim with the norm $S$ replaced by the norm $X$ in (3.5).

Next, an application of Lemma 2.2 along with (3.1), (3.4), and (3.11) yields

$$
\begin{aligned}
\|\tilde{u}\|_{S^{s_{c}(I)}} & \lesssim\left\|\nabla_{t, x} \tilde{u}\right\|_{L_{t}^{\infty} \dot{H}_{x}^{s_{c}-1}}+\left\||\nabla|^{s_{c}-\frac{1}{2}} e\right\|_{L_{t, x}^{\frac{2(d+1)}{d+3}}}+\left\||\nabla|^{s_{c}-\frac{1}{2}} F(\tilde{u})\right\|_{L_{t, x}^{\frac{2(d+1)}{d+3}}} \\
& \lesssim M+\varepsilon+\delta^{p}\|\tilde{u}\|_{S^{s_{c}(I)}} .
\end{aligned}
$$


Hence, for $\delta$ sufficiently small depending on $d, p$ and for $\varepsilon$ small enough depending on $M$,

$$
\|\tilde{u}\|_{S^{s_{c}(I)}} \lesssim M
$$

We first explain how to complete the argument in the case when $3 \leq d \leq 6$. Note that in this case, the power $p$ under discussion satisfies $p \geq 1$. An application of the Strichartz inequality Lemma 2.2 along with Lemma 3.3, (3.3), (3.4), (3.11), and (3.12) yields

$$
\begin{aligned}
\|u-\tilde{u}\|_{S(I)}+ & \|u-\tilde{u}\|_{X(I)} \\
\lesssim & \left\|\left(u_{0}, u_{1}\right)-\left(\tilde{u}_{0}, \tilde{u}_{1}\right)\right\|_{\dot{H}_{x}^{s_{c}} \times \dot{H}_{x}^{s_{c}-1}}+\left\||\nabla|^{s_{c}-\frac{1}{2}} e\right\|_{L_{t, x}^{\frac{2(d+3)}{d+3}}} \\
& +\left\||\nabla|^{s_{c}-\frac{1}{2}}[F(u)-F(\tilde{u})]\right\|_{L_{t, x}^{\frac{2(d+3)}{d+3}}} \\
\lesssim & \varepsilon+\|\tilde{u}\|_{X(I)}\|u-\tilde{u}\|_{S(I)}^{p}+\|u-\tilde{u}\|_{X(I)}\left\{\|\tilde{u}\|_{S(I)}^{p}+\|u-\tilde{u}\|_{S(I)}^{p}\right\} \\
\lesssim & \varepsilon+\delta^{\tilde{\theta}} M^{1-\tilde{\theta}}\|u-\tilde{u}\|_{S(I)}^{p}+\delta^{p}\|u-\tilde{u}\|_{X(I)}+\|u-\tilde{u}\|_{X(I)}\|u-\tilde{u}\|_{S(I)}^{p} .
\end{aligned}
$$

Taking $\delta$ small depending on $d, p, M$ and recalling that $p \geq 1$, a bootstrap argument yields

$$
\|u-\tilde{u}\|_{S(I)}+\|u-\tilde{u}\|_{X(I)} \lesssim \varepsilon
$$

which settles (3.5). Another application of the Strichartz inequality as above proves (3.6). The estimate (3.7) follows from the triangle inequality, (3.6), and (3.12).

We now consider the case when $d \geq 7$. Arguing as in the main estimate for the lower-dimensional case, but now using Lemma 3.2 as well, we obtain

$$
\begin{aligned}
\|u-\tilde{u}\|_{X(I)} & \\
& \lesssim\left\|\left(u_{0}, u_{1}\right)-\left(\tilde{u}_{0}, \tilde{u}_{1}\right)\right\|_{\dot{H}_{x}^{s_{c}} \times \dot{H}_{x}^{s_{c}-1}}+\left\||\nabla|^{s_{c}-\frac{1}{2}} e\right\|_{L_{t, x}^{\frac{2(d+1)}{d+3}}+\|F(u)-F(\tilde{u})\|_{Y(I)}} \\
& \lesssim \varepsilon+\|u-\tilde{u}\|_{X(I)}\left\{\|u-\tilde{u}\|_{S^{s_{c}(I)}}^{p-\frac{p}{d}}\|u-\tilde{u}\|_{S(I)}^{\frac{p}{d}}+\|\tilde{u}\|_{S^{s_{c}(I)}}^{p-\frac{p}{d}}\|\tilde{u}\|_{S(I)}^{\frac{p}{d}}\right\} \\
\quad & \lesssim \varepsilon+\|u-\tilde{u}\|_{X(I)}^{1+\theta \frac{p}{d}}\|u-\tilde{u}\|_{S^{s_{c}(I)}}^{p-\theta \frac{p}{d}}+\delta^{\frac{p}{d}} M^{p-\frac{p}{d}}\|u-\tilde{u}\|_{X(I)},
\end{aligned}
$$

which for $\delta$ small (depending on $d, p, M$ ) yields

$$
\|u-\tilde{u}\|_{X(I)} \lesssim \varepsilon+\|u-\tilde{u}\|_{X(I)}^{1+\theta \frac{p}{d}}\|u-\tilde{u}\|_{S^{s_{c}}(I)}^{p-\theta \frac{p}{d}} .
$$

Another application of the same considerations (using (3.12) ) yields

$$
\begin{aligned}
\| u-\tilde{u} & \|_{S^{s_{c}}(I)} \\
& \lesssim \\
& \quad\left\|\left(u_{0}, u_{1}\right)-\left(\tilde{u}_{0}, \tilde{u}_{1}\right)\right\|_{\dot{H}_{x}^{s_{c}} \times \dot{H}_{x}^{s_{c}-1}}+\left\||\nabla|^{s_{c}-\frac{1}{2}} e\right\|_{L_{t, x}^{\frac{2(d+3)}{d+3}}} \\
& +\left\|\left.\nabla\right|^{s_{c}-\frac{1}{2}}[F(u)-F(\tilde{u})]\right\|_{L_{t, x}^{\frac{2(d+1)}{d+3}}} \\
& \lesssim \varepsilon+\|\tilde{u}\|_{S^{s_{c}(I)}}\|u-\tilde{u}\|_{S(I)}^{p}+\|u-\tilde{u}\|_{S^{s_{c}(I)}}\left\{\|u-\tilde{u}\|_{S(I)}^{p}+\|\tilde{u}\|_{S(I)}^{p}\right\} \\
& \lesssim \varepsilon+\left[M\|u-\tilde{u}\|_{S^{s_{c}(I)}}^{(1-\theta) p}+\|u-\tilde{u}\|_{S^{s_{c}(I)}}^{1+(1-\theta) p}\right]\|u-\tilde{u}\|_{X(I)}^{\theta p}+\delta^{p}\|u-\tilde{u}\|_{S^{s_{c}(I)}},
\end{aligned}
$$

which for $\delta$ small (depending on $d, p$ ) gives

$$
\|u-\tilde{u}\|_{S^{s_{c}(I)}} \lesssim \varepsilon+\left[M\|u-\tilde{u}\|_{S^{s_{c}(I)}}^{(1-\theta) p}+\|u-\tilde{u}\|_{S^{s_{c}(I)}}^{1+(1-\theta) p}\right]\|u-\tilde{u}\|_{X(I)}^{\theta p} .
$$


A simple bootstrap argument using (3.13) and (3.14) yields (3.5) and (3.6). The claim (3.7) follows by the triangle inequality, (3.6), and (3.12). This completes the proof of the theorem.

\section{The GLOBAL SOLUTiONS}

In this section we preclude the soliton-like and frequency-cascade solutions described in Theorem 1.7 .

Theorem 4.1 (Absence of solitons and frequency-cascades). There are no solitonlike or frequency-cascade solutions to (1.1) in the sense of Theorem 1.7.

Proof. We argue by contradiction. Assume there exists a solution $u: \mathbb{R} \times \mathbb{R}^{d} \rightarrow \mathbb{R}$ that is either a soliton-like or a frequency-cascade solution in the sense of Theorem 1.7. We will show these scenarios are inconsistent with the Morawetz inequality.

By Lemma 2.5.

$$
\int_{0}^{T} \int_{|x| \leq T} \frac{|u(t, x)|^{p+2}}{|x|} d x d t \lesssim u T^{d-2-\frac{4}{p}}
$$

for any $T>0$. On the other hand, by Proposition 2.6 we have concentration of potential energy, that is, there exists $C=C(u)$ so that for any $T \geq N(0)^{-1} \geq 1$,

$$
\int_{0}^{T} \int_{|x| \leq C / N(t)} N(t)|u(t, x)|^{p+2} d x d t \gtrsim u \int_{0}^{T} N(t)^{\frac{4}{p}-(d-3)} d t .
$$

Using the fact that $N(t) \geq 1$ and $p<\frac{4}{d-3}$, we obtain that for $T \geq 1$,

$$
\operatorname{LHS(4.1)} \geq \int_{0}^{T} \int_{|x| \leq C / N(t)} \frac{|u(t, x)|^{p+2}}{|x|} d x d t \gtrsim_{u} \int_{0}^{T} N(t)^{\frac{4}{p}-(d-3)} d t \gtrsim_{u} T .
$$

Choosing $T$ sufficiently large depending on $u$ and recalling once again that $p<\frac{4}{d-3}$ (and hence $\frac{4}{p}-(d-2)<1$ ), we derive a contradiction to (4.1).

\section{The Finite-time BlOW-UP SOLUtion}

In this section, we preclude the finite-time blow-up scenario described in Theorem 1.7 by showing that such solutions are inconsistent with the conservation of energy.

Theorem 5.1 (Absence of finite-time blow-up solutions). There are no finite-time blow-up solutions to (1.1) in the sense of Theorem 1.7.

Proof. We argue by contradiction. Assume there exists a solution $u: I \times \mathbb{R}^{d} \rightarrow \mathbb{R}$ that is a finite-time blow-up solution in the sense of Theorem 1.7. By the timereversal and time-translation symmetries, we may assume that the solution blows up as $t \searrow 0=\inf I$.

First note that $N(t) \rightarrow \infty$ as $t \rightarrow 0$, for otherwise any subsequential limit of the functions $N(t)^{-\frac{2}{p}} u\left(t, N(t)^{-1} x\right)$ would blow up instantaneously, in contradiction to the local theory.

Next we show that

$$
\operatorname{supp} u(t) \cup \operatorname{supp} u_{t}(t) \subseteq B(0, t) \quad \text { for all } t \in I,
$$


where $B(0, t)$ denotes the closed ball in $\mathbb{R}^{d}$ centered at the origin of radius $t$. Indeed, it suffices to show that

$$
\lim _{t \rightarrow 0} \int_{t+\varepsilon \leq|x| \leq \varepsilon^{-1}-t} \frac{1}{2}\left|\nabla_{t, x} u(t, x)\right|^{2}+\frac{1}{p+2}|u(t, x)|^{p+2} d x=0 \quad \text { for all } \quad \varepsilon>0
$$

because the energy on the annulus $\left\{x: t+\varepsilon \leq|x| \leq \varepsilon^{-1}-t\right\}$ is finite and does not decrease as $t \rightarrow 0$. To obtain (5.2), fix $\varepsilon>0$. As $N(t) \rightarrow \infty$ as $t \rightarrow 0$, we deduce that for all $\eta>0$ there exists $t_{0}=t_{0}(\varepsilon, \eta)$ such that for $0<t<t_{0}$ we have

$$
\left\{x \in \mathbb{R}^{d}: t+\varepsilon \leq|x| \leq \varepsilon^{-1}-t\right\} \subseteq\left\{x \in \mathbb{R}^{d}:|x| \geq C(\eta) / N(t)\right\},
$$

where $C(\eta)$ is as in (1.6). Thus by Hölder's inequality and (1.6),

$$
\begin{aligned}
& \int_{t+\varepsilon \leq|x| \leq \varepsilon^{-1}-t}\left(\frac{1}{2}\left|\nabla_{t, x} u(t, x)\right|^{2}+\frac{1}{p+2}|u(t, x)|^{p+2}\right) d x \\
& \quad \lesssim \varepsilon^{\frac{4}{p}-(d-2)}\left[\left\|\nabla_{t, x} u(t)\right\|_{L_{x}^{\frac{d p}{p+2}}(\{|x| \geq C(\eta) / N(t)\})}^{2}+\|u(t)\|_{L_{x}^{\frac{d p}{2}}(\{|x| \geq C(\eta) / N(t)\})}^{p+2}\right] \\
& \quad \lesssim \varepsilon^{\frac{4}{p}-(d-2)} \eta^{2}
\end{aligned}
$$

for all $0<t<t_{0}$. As $\eta$ can be made arbitrarily small, this proves (5.2) and hence (5.1).

To continue, by (5.1), Hölder's inequality, and Sobolev embedding we obtain

$$
\begin{aligned}
E(u(t)) & =\int_{B(0, t)}\left(\frac{1}{2}\left|\nabla_{t, x} u(t, x)\right|^{2}+\frac{1}{p+2}|u(t, x)|^{p+2}\right) d x \\
& \lesssim\left(\left\|\nabla_{t, x} u(t)\right\|^{2} \frac{d p}{L_{x}^{p+2}}+\|u(t)\|_{L_{x}^{\frac{d p}{2}}}^{p+2}\right) t^{d-2-\frac{4}{p}} \\
& \lesssim u t^{d-2-\frac{4}{p}}
\end{aligned}
$$

for all $t \in I$. In particular, the energy of the solution is finite and converges to zero as the time $t$ approaches the blow-up time 0. Invoking the conservation of energy, we deduce that $u \equiv 0$. This contradicts the fact that $u$ is a blow-up solution.

\section{REFERENCES}

[1] H. Bahouri and P. Gérard, High frequency approximation of solutions to critical nonlinear wave equations. Amer. J. Math. 121 (1999), 131-175. MR.1705001

[2] J. Bourgain, Global wellposedness of defocusing critical nonlinear Schrödinger equation in the radial case. J. Amer. Math. Soc. 12 (1999), 145-171. MR.1626257

[3] A. Bulut, Maximizers for the Strichartz inequalities for the wave equation. Preprint arXiv:0905.1678.

[4] A. Bulut, Global well-posedness and scattering for the defocusing energy-supercritical cubic nonlinear wave equation. Preprint arXiv:1006.4168

[5] A. Bulut, M. Czubak, D. Li, N. Pavlovic, X. Zhang, Stability and unconditional uniqueness of solutions for energy critical wave equations in high dimensions. Preprint arXiv:0911.4534.

[6] J. Ginibre, A. Soffer, and G. Velo, The global Cauchy problem for the critical nonlinear wave equation. J. Funct. Anal. 110 (1992), 96-130. MR.1190421

[7] M. Grillakis, Regularity and asymptotic behaviour of the wave equation with a critical nonlinearity. Ann. of Math. (2) 132 (1990), 485-509. MR.1078267

[8] M. Grillakis, Regularity for the wave equation with a critical nonlinearity. Comm. Pure Appl. Math. 45 (1992), 749-774. MR1162370 (93e:35073)

[9] L. Kapitanskii, The Cauchy problem for the semilinear wave equation. I, II, III. Zap. Nauchn. Sem. Leningrad. Otdel. Mat. Inst. Steklov. (LOMI) 163 (1987), 76-104; 182 (1990), 38-85; and 181 (1990), 24-64. MR0918943 MR.1064097 MR.1097579 
[10] M. Keel and T. Tao, Endpoint Strichartz estimates. Amer. J. Math. 120 (1998), 955-980. MR:1646048

[11] C. E. Kenig and F. Merle, Global well-posedness, scattering and blow-up for the energycritical, focusing, non-linear Schrödinger equation in the radial case. Invent. Math. 166 (2006), 645-675. MR2257393

[12] C. E. Kenig and F. Merle, Global well-posedness, scattering and blow-up for the energy-critical focusing non-linear wave equation. Acta Math. 201 (2008), 147-212. MR2461508

[13] C. E. Kenig and F. Merle, Nondispersive radial solutions to energy supercritical non-linear wave equations, with applications. Preprint arXiv:0810.4834.

[14] S. Keraani, On the blow-up phenomenon of the critical nonlinear Schrödinger equation. J. Funct. Anal. 235 (2006), 171-192. MR.2216444

[15] R. Killip and M. Visan, The focusing energy-critical nonlinear Schrödinger equation in dimensions five and higher. Amer. J. Math. 132 (2010), 361-424. MR2654778

[16] R. Killip and M. Visan, Nonlinear Schrödinger equations at critical regularity. Lecture notes prepared for Clay Mathematics Institute Summer School, Zürich, Switzerland, 2008.

[17] R. Killip and M. Visan, Energy-supercritical NLS: critical $H^{s}$-bounds imply scattering. Preprint arXiv:0812.2084. To appear in Comm. Partial Differential Equations.

[18] R. Killip, M. Visan, and X. Zhang, Energy-critical NLS with quadratic potentials. Comm. Partial Differential Equations 34 (2009), 1531-1565. MR2581982

[19] R. Killip and M. Visan, The defocusing energy-supercritical nonlinear wave equation in three space dimensions. Preprint arXiv:1001.1761 To appear in Trans. Amer. Math. Soc.

[20] C. S. Morawetz, Notes on time decay and scattering for some hyperbolic problems. Regional Conference Series in Applied Mathematics, No. 19. Society for Industrial and Applied Mathematics, Philadelphia, PA, 1975. MR0492919

[21] C. S. Morawetz and W. A. Strauss, Decay and scattering of solutions of a nonlinear relativistic wave equation. Comm. Pure Appl. Math. 25 (1972), 1-31. MR0303097

[22] K. Nakanishi, Scattering theory for nonlinear Klein-Gordon equation with Sobolev critical power. Internat. Math. Res. Notices 1 (1999), 31-60. MR1666973

[23] H. Pecher, Nonlinear small data scattering for the wave and Klein-Gordon equation. Math. Z. 185 (1984), 261-270. MR0731347

[24] J. Rauch, I. The $u^{5}$ Klein-Gordon equation. II. Anomalous singularities for semilinear wave equations. In "Nonlinear partial differential equations and their applications". Collège de France Seminar, Vol. I (Paris, 1978/1979), pp. 335-364, Res. Notes in Math., 53, Pitman, Boston, Mass.-London, 1981. MR0631403

[25] J. Shatah and M. Struwe, Regularity results for nonlinear wave equations. Ann. of Math. (2) 138 (1993), 503-518. MR1247991

[26] J. Shatah and M. Struwe, Geometric wave equations. Courant Lecture Notes in Mathematics, 2. Courant Institute of Mathematical Sciences, New York; American Mathematical Society, Providence, RI, 1998. MR.1674843

[27] R. S. Strichartz, Restrictions of Fourier transforms to quadratic surfaces and decay of solutions of wave equations. Duke Math. J. 44 (1977), 705-714. MR0512086

[28] M. Struwe, Globally regular solutions to the $u^{5}$ Klein-Gordon equation. Ann. Scuola Norm. Sup. Pisa Cl. Sci. 15 (1988), 495-513. MR.1015805

[29] T. Tao, Global well-posedness and scattering for the higher-dimensional energy-critical non-linear Schrödinger equation for radial data. New York J. of Math. 11 (2005), 57-80. MR2154347

[30] T. Tao, Spacetime bounds for the energy-critical nonlinear wave equation in three spatial dimensions. Dynamics of Partial Differential Equations 3 (2006), 93-110. MR2227039

[31] M. E. Taylor, Tools for PDE. Mathematical Surveys and Monographs, 81. American Mathematical Society, Providence, RI, 2000. MR1766415

[32] M. Visan, The defocusing energy-critical nonlinear Schrödinger equation in higher dimensions. Duke Math. J. 138 (2007), 281-374. MR2318286

Department of Mathematics, University of California, los Angeles, California 90095-1555

Department of Mathematics, University of California, los Angeles, California 90095-1555 